\title{
Multiple Chronic Conditions and Functional Outcome after Ischemic Stroke: A Systematic Review and Meta-Analysis
}

\author{
Xiaqing Jiang ${ }^{a} \quad$ Lewis B. Morgenstern $^{a, b} \quad$ Christine T. Cigolle ${ }^{c-e}$ \\ Edward S. Claflin ${ }^{f}$ Lynda D. Lisabeth ${ }^{a, b}$ \\ ${ }^{a}$ Department of Epidemiology, School of Public Health, University of Michigan, Ann Arbor, MI, USA; ${ }^{b}$ Stroke \\ Program, University of Michigan Medical School, Ann Arbor, MI, USA; ' Department of Family Medicine, University \\ of Michigan Medical School, Ann Arbor, MI, USA; ${ }^{d}$ Department of Internal Medicine, University of Michigan Medical

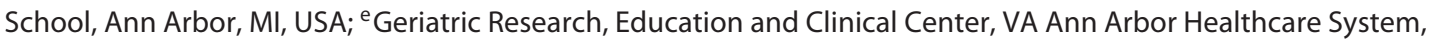

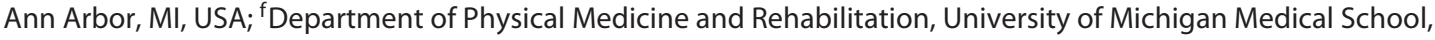 \\ Ann Arbor, MI, USA
}

\section{Keywords}

Multiple chronic conditions · Multimorbidity · Stroke ·

Patient outcome assessment · Disability

\begin{abstract}
Background: Multiple chronic conditions (MCC) contribute to functional disability in the general population although its role in predicting functional outcome (FO) among patients with stroke is not well understood. There is no universal agreement on the approach to measuring MCC in stroke, and findings have been mixed regarding MCC being an independent predictor for poststroke FO. Objectives: This review aims to summarize the findings of studies that have investigated the relationship between MCC and FO after ischemic stroke using a MCC index. Method: PubMed and Embase were systematically searched for studies conducted among ischemic stroke patients that have examined the adjusted association between prestroke MCC and FO. The quality of the included studies was appraised using a risk of bias (RoB) assessment checklist. A meta-analysis was performed for the association between MCC and FO using a random effects model to estimate the overall pooled ORs. Results: Twelve of the 18 studies included were hospital-based co-
\end{abstract}

() 2019 S. Karger AG, Basel hort studies, with a median RoB score of 4.75 points (range 1-9, higher scores for higher RoB). Studies predominantly used the Charlson Comorbidity Index (CCl), or the Modified $\mathrm{CCl}$ to measure MCC burden, and the modified Rankin scale to measure FO. Half of the studies reported a significant negative association between MCC and FO, which was also found by the meta-analysis with a pooled OR of $1.11(95 \% \mathrm{Cl}$ 1.05-1.18). Conclusions: The current review supports that increased MCC is associated with worse poststroke FO although population-based studies of this association are lacking. Future research should aim to develop more refined measures of MCC that consider the severity and interactions of comorbid conditions reflective of the broader stroke population and to understand the relationship between MCC and poststroke FO with thorough adjustment for confounding factors.

(c) 2019 S. Karger AG, Basel

\section{Introduction}

Stroke is the 2nd leading cause of death and the 3rd leading cause of disability worldwide $[1,2]$. About $37-$ $45 \%$ of stroke survivors are functionally dependent at

\section{KARGER}

karger@karger.com

www.karger.com/ned
Lynda D. Lisabeth, $\mathrm{PhD}$

University of Michigan

Department of Epidemiology

1415 Washington Heights, Ann Arbor, MI 48109 (USA)

E-Mailllisabet@umich.edu 
1 year after stroke [3-5]. More than $30 \%$ of stroke survivors are dependent in one or more activities of daily living (ADLs), such as feeding, dressing, bathing, grooming, toileting, transfers, and mobility [6]. Limitations in ADLs are associated with decreased quality of life and increased use of hospital and nursing home care $[7,8]$. With the aging of the population and declining stroke mortality across the globe, there will be more stroke survivors coping with functional disability $[2,9]$. Ischemic stroke is the most prevalent stroke type, representing about 82 and $67 \%$ of all stroke events in high- and lowto-middle-income countries, respectively $[2,10]$. In the last 2 decades, thrombolytic treatments, such as intravenous tissue plasminogen activator and endovascular therapy, have improved functional outcome (FO) after ischemic stroke, but are received by only a small minority of stroke patients [11]. FO in nonthrombolyzed stroke survivors has been largely unchanged [12]. Similarly, acute inpatient rehabilitation, which reduces longterm disability and enhances FO, is received by only a minority of stroke patients after the acute hospitalization [13].

The aging of the world's population will also lead to an increase in the prevalence of multiple chronic conditions (MCC) at stroke onset [14-16]. MCC can be defined as the "concurrent presence of 2 or more medically diagnosed diseases in the same individual" [17]. The presence of MCC contributes considerably to frailty and functional impairment [16], primarily through pathophysiological changes and organ-level impairments [18]. Older patients with MCC who are physically and/or cognitively frail before stroke often have: (1) poorer cardiovascular and neuromuscular reserve due to decreased prestroke physical activity [19]; (2) worse hemodynamics and collateralization after arterial occlusions that impedes poststroke functional recovery [19]; and (3) higher stroke severity with increased risk of poststroke cognitive decline that hinders neuropsychological adaption after stroke [20-23]. In sum, MCC is considered to play an important role in diminishing the prestroke "reserve" that aids the neuropsychological process of poststroke adaption and recovery.

A modest association between MCC and poststroke FO in patients from inpatient rehabilitation was reported in a recent literature review [24]; however, this patient population is not a representative sample of the stroke patient population. The role of MCC in predicting FO in the broader stroke population is less well understood. In fact, although negative associations have been found, some studies showed that MCC was not independently associated with poststroke $\mathrm{FO}$ when other important predictors (e.g., age and stroke severity) were adjusted for [25-27]. Therefore, this systematic review aims to summarize the findings in studies that have investigated the relationship between MCC and FO after ischemic stroke.

\section{Materials and Methods}

This study was conducted in accordance with the recommendations of the Preferred Reporting Items for Systematic Reviews and Meta-Analysis Statement [28]. The authors declare that all supporting data are available within the article and in the online supplement Data (for all online suppl. material, see www.karger. com/doi/10.1159/000503900).

\section{Study Identification}

A systematic search of publications from January 1, 1990, to December 3, 2017, was carried out in the PubMed and Embase databases for relevant studies by the first author. The following keywords were used with their synonyms or similar terms in the search: ("stroke," or "cerebrovascular accident,") and ("MCCs," or "multimorbidity," or "comorbidity," or "comorbid conditions," or "predict," or "adjust,") and ("FO," or "functional independence," or "ADL," or "functional limitation," or "functional disability," or "functional recovery," or "Rankin," or "Functional Independence Measure (FIM)," or "modified Rankin scale (mRS)," or "Barthel”). The search strategies (online suppl. Table 1) were developed in conjunction with an experienced medical research librarian. MCCs are often included in prognostic modeling as an adjustment factor, and, therefore, restriction on studies mentioning terms related to MCC or comorbidity may exclude studies that did not focus on but included MCC in their analysis. For this reason, the search strategies aimed to find studies that used multivariable modeling to predict FO, which potentially included a MCC index as one of the covariates.

\section{Selection Criteria}

Studies were included if they satisfied the following criteria: (1) included adult ischemic stroke patients; (2) used a statistical method to analyze factors that are associated with FO; (3) used a global measurement of poststroke FO; (4) used a cumulative measurement of MCC; (5) reported associations between MCC and FO; and (6) were published in English.

Studies were excluded if they met any of the following criteria: (1) conducted only in patients who had hemorrhagic stroke, transient ischemic attack (TIA), or stroke types other than ischemic stroke; or (2) MCC was assessed dichotomously (with/without multiple conditions) or as a count of the number of comorbid conditions; or (3) the adjusted association between MCC and FO was not reported; or (4) was a systematic or topic review, letter, case report, or guideline; or (5) secondary analysis of clinical trials or cross-sectional studies. The first author screened all studies identified from the systematic search following these inclusion and exclusion criteria. Studies with questionable inclusion/exclusion criteria were resolved by consultation with a second reviewer (L.D.L.). 


\section{Data Abstraction}

A detailed data abstraction plan was developed by 2 authors (X.J., L.D.L.) before the first author extracted the following characteristics for each study: first author, year of publication, characteristics of the study population (country, number of subjects, age, stroke types, recruitment settings), study design, FO assessment (instrument and timing), MCC assessment, MCC-FO associations (univariate, multivariable) measured by ORs, relative risks (RRs), correlation coefficients, and so on, covariates included in the multivariable model, and model performance.

\section{Quality Appraisal}

A 26-item checklist was developed to examine the methodological quality of the included studies based on the risk of bias (RoB) from 6 domains: study participation, study attrition, MCC measurement, outcome measurement, statistical analysis, and clinical performance. This checklist was first developed using the guidelines from the Quality In Prognosis Studies tool [29] and the framework for assessing internal validity discussed by Altman [30], both geared to assess the RoB in studies of prognostic factors. Items that have been previously used in the quality appraisal for stroke prognostic studies were also considered [31]. The final checklist was tailored to consider items specific to the assessment of MCC.

Online supplemental Table 2 shows the quality criteria, which were graded as low ( 0 points) to high RoB (1 point) with a few items including a level of medium $\operatorname{RoB}(0.5$ point). Items with insufficient information were assigned the high RoB (1 point). A total score ranging $0-26$ was calculated by summing points from the 26 items for each study, and a study scored $\leq 5$ points (approximately $<80 \%$ of the maximum score) was considered as a high-quality study.

\section{Analysis}

A meta-analysis was performed to synthesize the information on the association between MCC and FO using the DerSimonian and Laird random-effects model to calculate a pooled effect-size estimate of the log RRs or ORs [32]. Assuming that the effect-sizes and CIs reported by each study were based on a Wald-like test of the null hypothesis that the true RR or OR is equal to 1 , the bounds of CIs were converted into the standard errors for each study. For studies that did not report CIs, $p$ values were converted into the corresponding $\mathrm{z}$-values. The calculated log ORs or RRs and the $\mathrm{z}$ values were then used to calculate the standard errors. When $p$ values were only reported as falling below a certain threshold (e.g., $p<0.01)$ or said to be significant $(p<0.05)$, the cutoff value was then used as a conservative estimate of the true $p$ value. Subgroup analysis was performed by MCC index used and the definition of poor outcome. Heterogeneity between studies was assessed using the $t$ test, and the percentage of the variability in effect estimates due to heterogeneity between studies was reported using the index. Funnel plot and Egger's regression test [33] for small meta-analysis ( $<25$ studies) was used to assess the risk of publication bias. All statistical analyses were performed using $\mathrm{R}$ version 3.5.0.

\section{Results}

\section{Study Selection}

The electronic database search resulted in 10,491 records, with an additional 42 articles identified from other sources. After the duplicate records were removed, 7,247 articles were screened. Among the 48 articles that assessed the MCC-FO relationship using valid MCC indices, 30 studies were ineligible. The reasons for exclusion are shown using the Preferred Reporting Items for Systematic Reviews and Meta-Analysis flowchart (Fig. 1).

No population-based cohort study was identified. Among the 18 eligible cohort studies, 6 were conducted in inpatient rehabilitation settings [27, 34-38]. Patients recruited from inpatient rehabilitation cohorts only represent a subset of the overall stroke population in the severity of comorbid conditions, cognitive status, and poststroke functioning (e.g., ADLs, motor involvement) [27, 34-38]. Therefore, these studies were separately summarized (online suppl. Table 3), and the qualitative and quantitative analyses of this review focused on the 12 hospital-based cohort studies.

\section{Study Characteristics}

Characteristics of the hospital-based cohort studies are summarized in online supplemental Table 3. Among the 12 studies, 11 studies were prospective cohorts recruited during hospitalization [25, 39-48]. There was 1 US [39] and 1 Asian cohort [25], while the majority of cohorts were from European countries [40-49].

All studies were published in the last 10 years except for one [39], with the number of participants ranging from 131 to 2,289. The assessment of the MCC-FO relationship was restricted to ischemic stroke patients in 9 studies [25, 39, 40, 42-44, 46, 47, 49], while the rest were mixed cohorts that included hemorrhagic stroke or TIA patients in addition to ischemic stroke patients. Two studies excluded patients with previous stroke [25, 49]. One study was conducted in veterans [39]. In the majority of the studies, the mean/median age of the participants was between 70 and 75 years old [40-48].

\section{RoB Assessment}

The median RoB total score was 4.75 points for the hospital-based cohorts (range 1 [47] -9 points [42]). Seven of the 12 studies were of high quality with low RoB [40, $43-45,47-49]$. The scores of the included studies are detailed in online supplemental Table 4.

\section{Outcome Assessment}

The mRS measured at 1 [49], 3 [40, 41, 43, 44, 47], or 6 [45] months after stroke onset was the most commonly used measure for FO (6 out of 12 studies). Other FO measures, including the FIM [25], SF-12 physical functioning domain/component score [46], or the Barthel index [48], 
Fig. 1. Preferred reporting items for systematic reviews and meta-analysis flow chart of literature search. MCC, multiple chronic condition; FO, functional outcome.

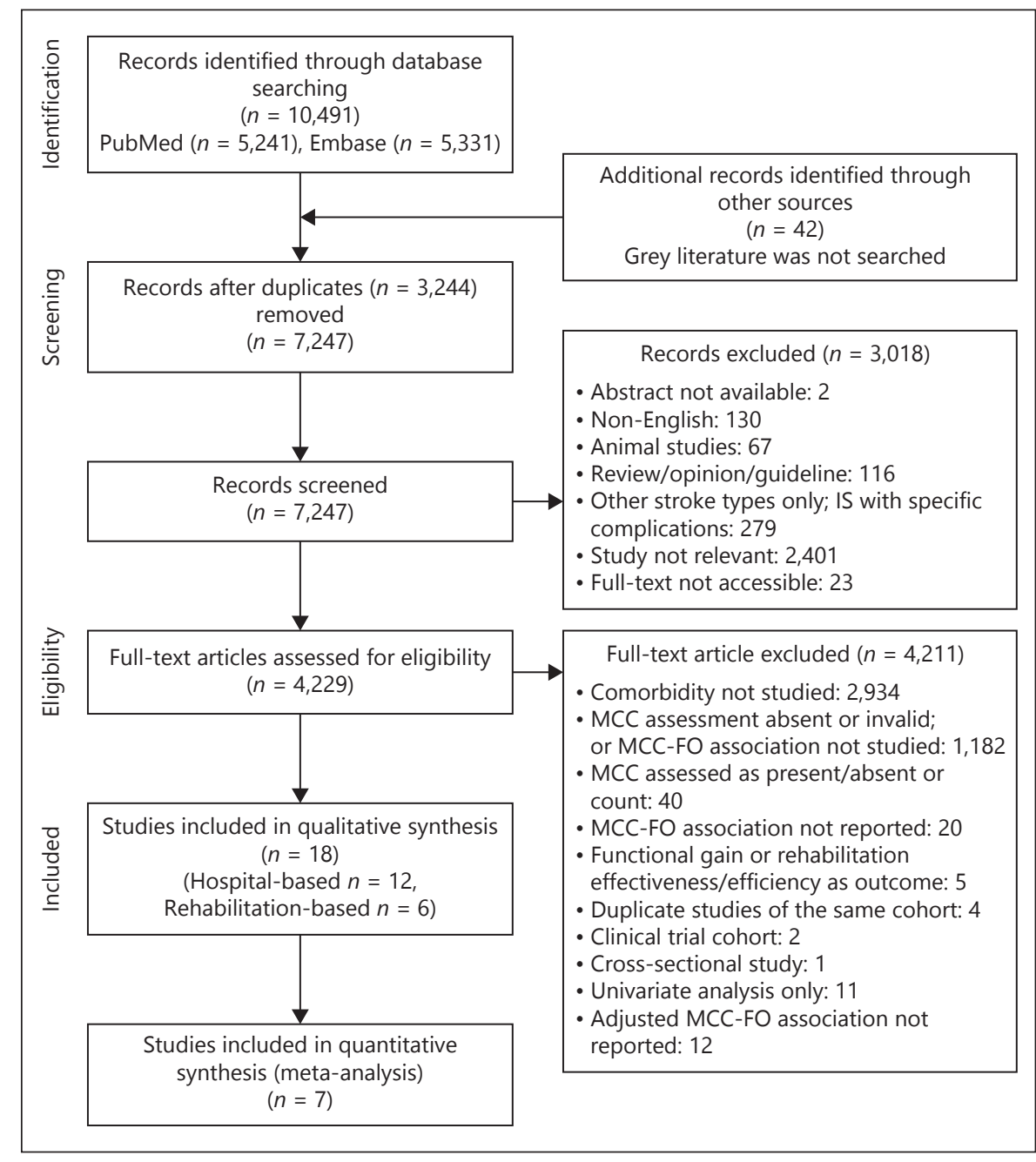

were also used. Two studies assessed FO at hospital discharge using mRS [39] or the number of impaired basic ADLs [42].

Among studies that assessed $\mathrm{mRS}$ at 3-6 months [40,41, $43-45,47]$, the proportion of patients with favorable outcome (mRS 0-2) was similar, ranging from 54.0 to $61.7 \%$, with the exception of one that included TIA patients and reported a higher proportion (68.7\%) [41]. Patients from these cohorts had similar baseline stroke severity (median National Institutes of Health Stroke Scale [NIHSS] 5-6) with the exception of 1 study that only included thrombolyzed patients (median NIHSS score of 14) [43].

\section{Assessment}

Two comorbidity indices were used in the hospitalbased studies. Half of the studies used the Charlson Comorbidity Index (CCI) [25, 40, 42, 45, 46, 48]. A stroke-adjusted version of the CCI or the Modified CCI (MCI) was used in the remaining studies. Briefly, CCI includes 19 chronic conditions weighted by their strength of associations with mortality [50]. CCI was originally developed as a prognostic indicator in patients with a variety of conditions and validated in breast cancer patients [50]. MCI is similar but excludes cerebrovascular disease and hemiplegia [39].

\section{Associations between MCC and FO}

The MCC-FO associations were investigated using multiple logistic regression and linear regression models, and the strength of multivariable associations was reported as ORs [25, 39-45, 47-49] or a regression coefficient ( $\beta$ ) [46]. All studies adjusted for age and stroke severity measured by NIHSS, with the exception of $2[42,46]$.

Among the 7 high-quality studies, 3 [40, 45, 47] of 5 studies that measured FO using mRS at 3-6 months found significant associations between MCC and poor FO, after adjusting for confounders including age and 


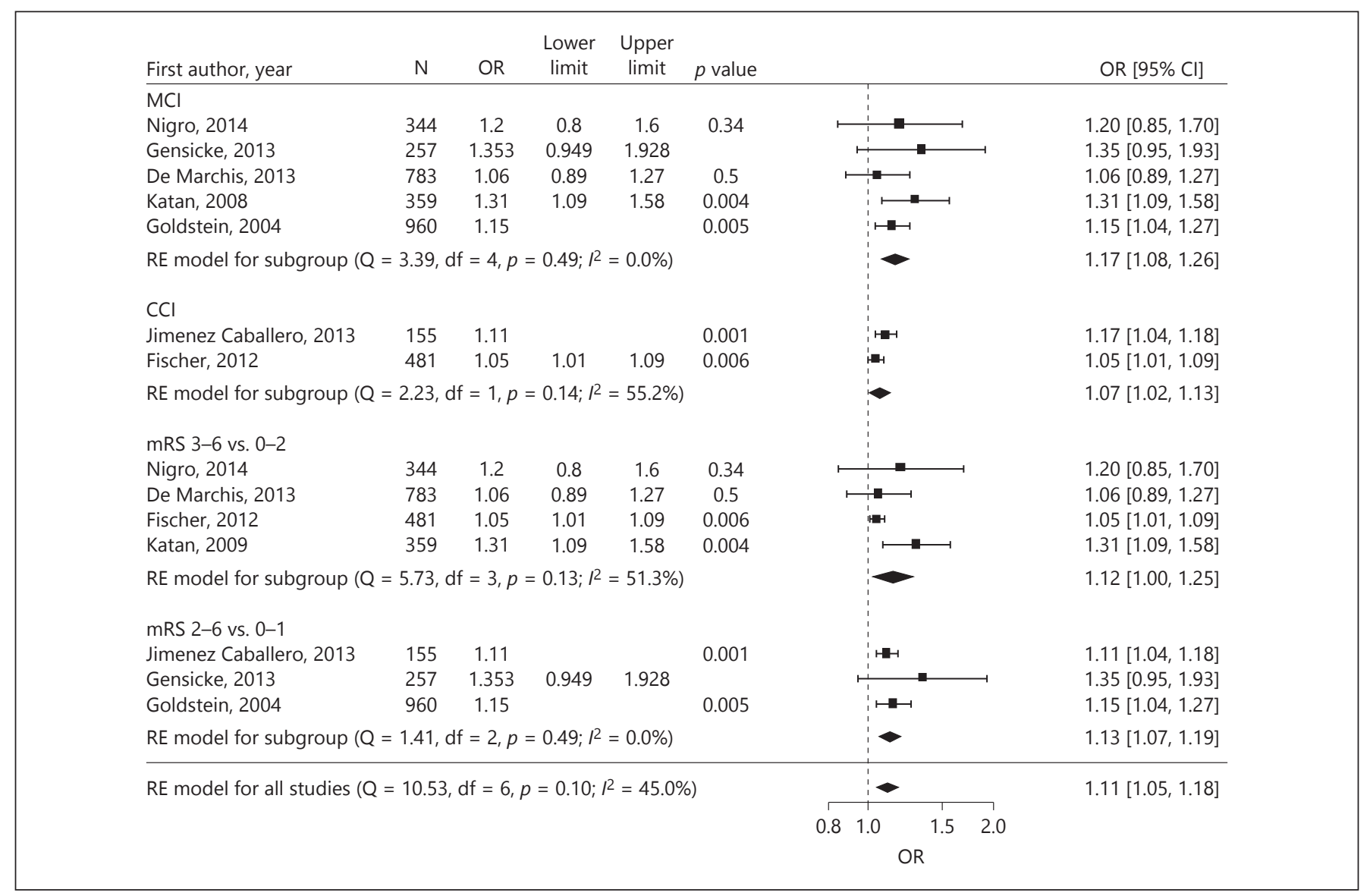

Fig. 2. Forest plot for ORs of poor FO. MCI, modified Charlson Comorbidity Index; CCI, Charlson Comorbidity Index; mRS, modified Rankin scale.

stroke severity. Specifically, the odds of poor FO were 5\% [40], 11\% [45], or 31\% [47] higher with every 1 point increase in CCI or MCI. The other 2 high-quality studies that measured mRS at 1 month or Barthel index at 6 months failed to find significant associations with MCI after adjusting for age and NIHSS [48, 49].

Among studies with lower methodological quality [25, $39,41,42,46]$, MCC was found to be significantly associated with poor FO measured by mRS [39] and ADL impairments [42] at hospital discharge; SF-12 at 6 months [46] but not FIM measured at 6 months [25]. Therefore, significant associations between MCC and poor FO after adjustment were reported in half of the hospital-based cohort studies.

\section{Data Synthesis and Meta-Analysis}

Significant heterogeneity existed among the studies in terms of study population, outcome, and MCC measure, as well as analytical methods and covariate adjustment for the multivariable models. Consequently, quantitative analysis was limited to 7 hospital-based cohorts that used similar MCC and outcome measures, namely, a continuous MCC measure using MCI/CCI and a dichotomized FO measure using mRS [39-41, 43-45, 47]. Five of the studies included in the meta-analysis were deemed highquality studies.

The pooled ORs overall and by subgroups are shown in Figure 2. MCC was significantly associated with poor FO (pooled OR 1.11; 95\% CI 1.05-1.18). In subgroup analysis, the pooled OR for studies using MCI was somewhat larger than that for studies using CCI (pooled OR 1.17 vs. 1.07). Associations were statistically significant in both subgroups defined by MCC measure. A significant association was found for studies that defined poor outcome as mRS 2-6 (pooled OR 1.13; 95\% CI 1.07-1.19) and for studies that defined poor outcome as mRS 3-6 (pooled OR 1.12; 95\% CI 1.00-1.25). Heterogeneity between the 7 studies was low to moderate, with $45 \%$ of the variability in ORs due to heterogeneity between studies 


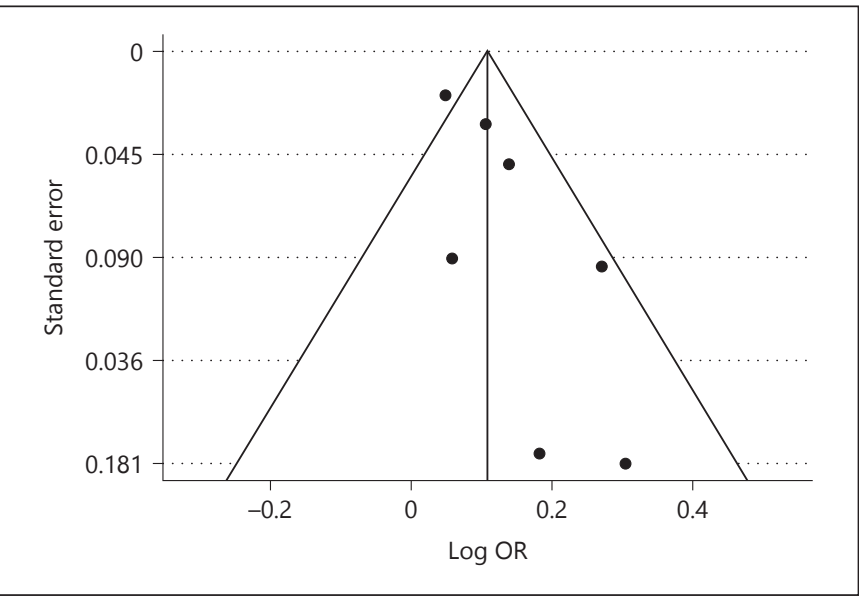

Fig. 3. Funnel plot for included studies.

(= 45\%, $p=0.1$ ). The funnel plot (Fig. 3) demonstrated some asymmetry, and the Egger's regression test showed evidence of publication bias $(p=0.0153)$.

\section{Discussion}

This review investigated the association between MCC and poststroke FO in the general stroke population. In the absence of population-based studies, the review focused on single and multicentered, hospital-based cohort studies. Two indices, CCI and MCI, were used to assess MCC burden in the examination of the multivariable adjusted MCC-FO association. Although the outcome assessment varied in terms of scale and timing of the assessment, half of the 12 included studies reported a significant association between increased MCC and worse FO. In our metaanalysis that included primarily high-quality studies with low to medium heterogeneity, a significant association between increased MCC and worse FO was also found. Therefore, the current review supports that MCC is negatively associated with poststroke FO.

Through our review, we identified gaps and limitations in the current literature regarding the association of MCC and poststroke FO, which could inform future research directions. Specifically, there is potential for improving the prediction of FO through the development of more refined MCC indices so that the impact of MCC can be assessed more accurately. Key areas for developing refined indices include: (1) consideration of a larger number of conditions with a focus on conditions relevant for function, (2) consideration of the possible synergistic effects of conditions, (3) consideration of the severity of conditions, (4) consid- eration of prestroke function, and (5) development of new indices in population-based stroke studies reflective of the broader stroke population. Limitations of the current research in each of these areas are briefly discussed.

The predominantly used MCC indices, CCI and MCI, consider a limited number of conditions originally included to predict mortality $[39,50]$, and the weights used reflect the degree of their impact on mortality rather than function [38]. Importantly, conditions that predict function in the elderly, including osteoarthritis/arthritis, hypertension, visual and hearing impairments, and atrial fibrillation, are not captured in CCI or MCI $[18,35]$. This could result in an observed smaller range of values in MCC indices, and thus a comparatively smaller proportion of variance in $\mathrm{FO}$ explained by MCC than that in the clinical reality.

It is known that certain combinations of conditions can have a synergistic effect on risk of disability [17, 51, 52]; however, these interactions among MCC are not captured by currently used MCC indices [17]. The development of novel MCC indices that include a greater number of conditions, as well as the possible synergistic interactions between conditions, would be challenging with conventional statistical methods, such as multivariable regression, due to the high dimensionality of the data. More contemporary methods, such as machine learning techniques, that have the ability of performing simultaneous model selection and estimation may be an ideal approach for developing more refined MCC indices in the future [53].

Neither CCI nor MCI consider the severity of conditions when measuring MCC burden. Previous research conducted with patients during acute rehabilitation found a stronger association between FO and MCC measures with severity weights compared to MCC measures without severity weights [54]. Inclusion of weights allows an index to quantify the difference in MCC burden driven by differences in severity levels of the conditions rather than condition types alone. Unfortunately, such indices have only been developed in select rehabilitation populations limiting their utility.

While studies examining the association between MCC and FO were often adjusted for age and stroke severity (NIHSS), prestroke function was less often considered in the analysis. Prestroke functional status largely defines the patients' poststroke FO. Prestroke cognitive and physical function play inseparable parts in the neuropsychological process of poststroke adaption and recovery [55]. Future research should consider patients' prestroke status in the analysis, or consider inclusion of prestroke functional and cognitive status as part of MCC as has been done in research of the general population 
[56]. To date, none of the currently used MCC indices were developed in population-based studies, which reflect the full spectrum of chronic conditions in the stroke population. Some indices were developed only in rehabilitation patients, which represent less than one-third of the total stroke population [13]. Because patients with more severe comorbid conditions are often excluded from rehabilitation, patients from inpatient rehabilitation have a different distribution and level of severity in comorbid conditions compared to the majority of stroke patients [35, 37]. Further, MCC indices used in rehabilitation studies often mix poststroke complications with prestroke comorbidities (e.g., the Liu comorbidity index) $[34,35,38]$, which limits the applicability of their findings to early FO prediction during acute stroke hospitalization when complications of stroke may still be evolving. Thus, new MCC indices developed and validated in populationbased studies with thorough adjustment for confounding factors are needed to minimize bias and better predict FO.

Another limitation of existing studies is the use of global disability measures, predominantly the mRS, which while useful for distinguishing broad categories of disability, does not measure function related to ADLs and is not sensitive to smaller differences in FO between patients [57]. For example, a patient who cannot walk independently scores 4 for mRS, whether or not he/she also needs help in eating, going to the toilet, and bathing. Dichotomizing the mRS score also adds to this insensitiveness of partial differences between patients (mRS 3 vs. 5) [58]. The mRS score does not measure poststroke impairments in cognitive function, language, vision, emotion, and pain, although these deficits could have a substantial impact on performing ADLs. Poststroke cognitive function is especially relevant to the success of stroke rehabilitation. With a growing number of stroke patients with cognitive impairment, measuring cognitive function is becoming a necessary part of poststroke FO assessment. In addition, using $\mathrm{mRS}$ to measure $\mathrm{FO}$ is a limitation due to its low inter-rater agreement, which can range from 0.25 to 0.74 depending on whether a structured interview is used [58]. Further, studies using mRS often include death in the definition of poor FO making it difficult to distinguish the impact of MCC on function versus mortality. More detailed measures of poststroke FO, including FIM, were seldom used in the hospital-based studies. Future research using more refined FO measures is likely to provide a more nuanced understanding of the links between MCC and FO in stroke patients.

Our study is not without limitations. First, as the funnel plot and the Egger's regression test indicated, publica- tion bias may exist in the included studies, where significant MCC-FO associations are more likely to be published, presented, and subsequently included in our meta-analysis. Second, we excluded studies that used present/absent or a count of the number of comorbid conditions as MCC measurement, the discussion of which is beyond the scope of this work. Third, we excluded studies conducted purely among other stroke types, such as intracerebral/subarachnoid hemorrhage and lacunar infarction. Therefore, caution should be taken generalizing our findings to patients with other types of stroke.

In conclusion, we found that greater prestroke MCC was associated with worse poststroke FO in hospital-based cohorts after adjusting for stroke severity and age. New MCC measures which capture conditions that are more relevant for predicting poststroke FO among stroke survivors are needed. A greater understanding of the association between MCC and poststroke FO could contribute to more personalized prognosis regarding FO, greater attention to the prevention and management of MCC, and better understanding of the specific rehabilitation needs of those with MCC to aid patients in the successful recovery of stroke.

\section{Acknowledgments}

None.

\section{Statement of Ethics}

The authors have no ethical conflicts to disclose.

\section{Disclosures Statement}

The authors have no conflicts of interest to declare.

\section{Funding Sources}

This work was supported by American Heart Association Predoctoral Fellowship 18PRE33990493/Xiaqing Jiang/2018.

\section{Author Contributions}

X.J., L.M., C.C., and L.D.L. designed the study. X.J. performed literature search, screening, analysis, and drafted the manuscript. X.J. and L.D.L. designed the inclusion, exclusion, and quality appraisal criteria. L.M., C.C., L.D.L., and E.C. provided input to the writing and modification of the manuscript. All authors reviewed the manuscript. 


\section{References}

1 World Health Organization. Disease burden and mortality estimates. WHO. 2019.

2 Benjamin EJ, Blaha MJ, Chiuve SE, Cushman M, Das SR, Deo R, et al. Heart disease and stroke statistics-2017 update: A report from the american heart association. Circulation. 2017 Mar; 135(10):e146-603.

3 Sennfält S, Norrving B, Petersson J, Ullberg T. Long-term survival and function after stroke. Stroke. 2018, Epub ahead of print.

4 Appelros P, Nydevik I, Viitanen M. Poor outcome after first-ever stroke: predictors for death, dependency, and recurrent stroke within the first year. Stroke. 2003 Jan;34(1):122-6.

5 Vuong Z. There's hope for reversing strokeinduced long-term disability. 2016-08-22 [cited 2019 June 1]; Available from: https://news. usc.edu/104649/theres-hope-for-reversingstroke-induced-long-term-disability/.

6 Dombovy ML, Basford JR, Whisnant JP, Bergstralh EJ. Disability and use of rehabilitation services following stroke in Rochester, Minnesota, 1975-1979. Stroke. 1987 Sep-Oct; 18(5):830-6.

7 Demaerschalk BM, Kleindorfer DO, Adeoye OM, Demchuk AM, Fugate JE, Grotta JC, et al.; American Heart Association Stroke Council and Council on Epidemiology and Prevention. Scientific rationale for the inclusion and exclusion criteria for intravenous alteplase in acute ischemic stroke: A statement for healthcare professionals from the american heart association/american stroke association. Stroke. 2016 Feb;47(2):581-641.

8 Matchar DB, Bilger M, Do YK, Eom K. International comparison of poststroke resource use: A longitudinal analysis in europe. J Stroke Cerebrovasc Dis. 2015 Oct;24(10):2256-62.

9 Feigin VL, Norrving B, Mensah GA. Global burden of stroke. Circ Res. 2017 Feb;120(3): 439-48.

10 Feigin VL, Lawes CM, Bennett DA, BarkerCollo SL, Parag V. Worldwide stroke incidence and early case fatality reported in 56 population-based studies: a systematic review. Lancet Neurol. 2009 Apr;8(4):355-69.

11 Sajobi TT, Zhang Y, Menon BK, Goyal M, Demchuk AM, Broderick JP, et al. Effect size estimates for the escape trial: proportional odds regression versus other statistical methods. Stroke. 2015 Jul;46(7):1800-5.

12 The National Institute of Neurological Disorders and Stroke rt-PA Stroke Study Group. Tissue plasminogen activator for acute ischemic stroke. N Engl J Med. 1995 Dec;333(24): 1581-7.

13 Morgenstern LB, Sais E, Fuentes M, Ifejika NL, Jiang X, Horn SD, et al. Mexican americans receive less intensive stroke rehabilitation than non-hispanic whites. Stroke. 2017 Jun;48(6):1685-7.

14 Kingston A, Robinson L, Booth H, Knapp M, Jagger C.; MODEM project. Projections of multi-morbidity in the older population in England to 2035: estimates from the Popula- tion Ageing and Care Simulation (PACSim) model. Age Ageing. 2018 May;47(3):374-80.

15 Buttorff C, Ruder T, Bauman M. Multiple chronic conditions in the united states. Santa Monica (CA): RAND Corporation; 2017. https://doi.org/10.7249/TL221.

16 U.S. Department of Health and Human Services. Multiple chronic conditions-a strategic framework: Optimum health and quality of life for individuals with multiple chronic conditions. Washington (DC): US Department of Health and Human Services; 2010.

17 Fried LP, Ferrucci L, Darer J, Williamson JD, Anderson G. Untangling the concepts of disability, frailty, and comorbidity: implications for improved targeting and care. J Gerontol A Biol Sci Med Sci. 2004 Mar;59(3):255-63.

18 Fried LP, Guralnik JM. Disability in older adults: evidence regarding significance, etiology, and risk. J Am Geriatr Soc. 1997 Jan; 45(1):92-100.

19 Stroud N, Mazwi TM, Case LD, Brown RD Jr, Brott TG, Worrall BB, et al.; Ischemic Stroke Genetics Study Investigators. Prestroke physical activity and early functional status after stroke. J Neurol Neurosurg Psychiatry. 2009 Sep;80(9):1019-22.

20 Reitz C, Bos MJ, Hofman A, Koudstaal PJ, Breteler MM. Prestroke cognitive performance, incident stroke, and risk of dementia: the Rotterdam Study. Stroke. 2008 Jan;39(1): 36-41.

21 Mysiw WJ, Beegan JG, Gatens PF. Prospective cognitive assessment of stroke patients before inpatient rehabilitation. The relationship of the Neurobehavioral Cognitive Status Examination to functional improvement. Am J Phys Med Rehabil. 1989 Aug;68(4):168-71.

22 Ozdemir F, Birtane M, Tabatabaei R, Ekuklu G, Kokino S. Cognitive evaluation and functional outcome after stroke. Am J Phys Med Rehabil. 2001 Jun;80(6):410-5.

23 Appelros P, Nydevik I, Seiger A, Terént A. Predictors of severe stroke: influence of preexisting dementia and cardiac disorders. Stroke. 2002 Oct;33(10):2357-62.

24 Kabboord AD, van Eijk M, Fiocco M, van Balen R, Achterberg WP. Assessment of comorbidity burden and its association with functional rehabilitation outcome after stroke or hip fracture: A systematic review and metaanalysis. J Am Med Dir Assoc. 2016 Nov; 17(11):1066.e13-21.

25 Chang WH, Sohn MK, Lee J, Kim DY, Lee SG, Shin YI, et al. Predictors of functional level and quality of life at 6 months after a firstever stroke: the KOSCO study. J Neurol. 2016 Jun;263(6):1166-77.

26 Fischer U, Arnold M, Nedeltchev K, Schoenenberger RA, Kappeler L, Höllinger P, et al. Impact of comorbidity on ischemic stroke outcome. Acta Neurol Scand. 2006 Feb;113(2):108-13.

27 Duncan PW, Horner RD, Reker DM, Samsa GP, Hoenig H, Hamilton B, et al. Adherence to postacute rehabilitation guidelines is associated with functional recovery in stroke. Stroke. 2002 Jan;33(1):167-77.

28 Shamseer L, Moher D, Clarke M, Ghersi D, Liberati A, Petticrew M, et al.; PRISMA-P Group. Preferred reporting items for systematic review and meta-analysis protocols (PRISMA-P) 2015: elaboration and explanation. BMJ. 2015 Jan 2;350:g7647.

29 Hayden JA, van der Windt DA, Cartwright JL, Côté P, Bombardier C. Assessing bias in studies of prognostic factors. Ann Intern Med. 2013 Feb; 158(4):280-6.

30 Altman DG. Systematic reviews of evaluations of prognostic variables. BMJ. $2001 \mathrm{Jul}$; 323(7306):224-8

31 Veerbeek JM, Kwakkel G, van Wegen EE, Ket JC, Heymans MW. Early prediction of outcome of activities of daily living after stroke: a systematic review. Stroke. 2011 May;42(5): 1482-8.

32 DerSimonian R, Laird N. Meta-analysis in clinical trials. Control Clin Trials. 1986 Sep; 7(3):177-88

33 Egger M, Davey Smith G, Schneider M, Minder C. Bias in meta-analysis detected by a simple, graphical test. BMJ. 1997 Sep;315(7109): 629-34.

34 Karatepe AG, Gunaydin R, Kaya T, Turkmen G. Comorbidity in patients after stroke: impact on functional outcome. J Rehabil Med. 2008 Nov;40(10):831-5.

35 Ferriero G, Franchignoni F, Benevolo E, Ottonello M, Scocchi M, Xanthi M. The influence of comorbidities and complications on discharge function in stroke rehabilitation inpatients. Eura Medicophys. 2006 Jun;42(2):91-6.

36 Desrosiers J, Noreau L, Rochette A, Bravo G, Boutin C. Predictors of handicap situations following post-stroke rehabilitation. Disabil Rehabil. 2002 Oct;24(15):774-85

37 Desrosiers J, Noreau L, Rochette A, Bourbonnais D, Bravo G, Bourget A. Predictors of long-term participation after stroke. Disabil Rehabil. 2006 Feb;28(4):221-30.

38 Liu M, Domen K, Chino N. Comorbidity measures for stroke outcome research: a preliminary study. Arch Phys Med Rehabil. 1997 Feb;78(2):166-72.

39 Goldstein LB, Samsa GP, Matchar DB, Horner RD. Charlson Index comorbidity adjustment for ischemic stroke outcome studies. Stroke. 2004 Aug;35(8):1941-5.

40 Fischer U, Mono ML, Zwahlen M, Nedeltchev K, Arnold M, Galimanis A, et al.; QABE investigators. Impact of thrombolysis on stroke outcome at 12 months in a population: the Bern stroke project. Stroke. 2012 Apr;43(4): 1039-45.

41 Nigro N, Wildi K, Mueller C, Schuetz P, Mueller B, Fluri F, et al. BNP but Not s-cTnln is associated with cardioembolic aetiology and predicts short and long term prognosis after cerebrovascular events. PLoS One. 2014 Jul;9(7):e102704. 
42 Tuttolomondo A, Di Raimondo D, Di Sciacca R, Pedone C, La Placa S, Arnao V, et al. Effects of clinical and laboratory variables at admission and of in-hospital treatment with cardiovascular drugs on short term prognosis of ischemic stroke. The GIFA study. Nutr Metab Cardiovasc Dis. 2013 Jul; 23(7):642-9.

43 Gensicke H, Seiffge DJ, Polasek AE, Peters N, Bonati LH, Lyrer PA, et al. Long-term outcome in stroke patients treated with IV thrombolysis. Neurology. 2013 Mar;80(10): 919-25.

44 De Marchis GM, Katan M, Weck A, Fluri F, Foerch C, Findling O, et al. Copeptin adds prognostic information after ischemic stroke: results from the CoRisk study. Neurology. 2013 Apr;80(14):1278-86.

45 Jimenez Caballero PE, Lopez Espuela F, Portilla Cuenca JC, Ramirez Moreno JM, Pedrera Zamorano JD, Casado Naranjo I. Charlson comorbidity index in ischemic stroke and intracerebral hemorrhage as predictor of mortality and functional outcome after 6 months. J Stroke Cerebrovasc Dis. 2013 Oct;22(7):e214-8.

46 Lopez-Espuela F, Zamorano JD, RamírezMoreno JM, Jiménez-Caballero PE, PortillaCuenca JC, Lavado-García JM, et al. Determi- nants of quality of life in stroke survivors after 6 months, from a comprehensive stroke unit: A longitudinal study. Biol Res Nurs. 2015 Oct; 17(5):461-8

47 Katan M, Fluri F, Morgenthaler NG, Schuetz P, Zweifel C, Bingisser R, et al. Copeptin: a novel, independent prognostic marker in patients with ischemic stroke. Ann Neurol. 2009 Dec;66(6):799-808.

48 Lopez-Espuela F, Pedrera-Zamorano JD, Jimenez-Caballero PE, Ramirez-Moreno JM, Portilla-Cuenca JC, Lavado-Garcia JM, et al. Functional status and disability in patients after acute stroke: A longitudinal study. Am J Crit Care. 2016 Mar;25(2):144-51.

49 Denti L, Artoni A, Casella M, Giambanco F, Scoditti U, Ceda GP. Validity of the modified charlson comorbidity index as predictor of short-term outcome in older stroke patients. J Stroke Cerebrovasc Dis. 2015 Feb;24(2): 330-6.

50 Charlson ME, Pompei P, Ales KL, MacKenzie CR. A new method of classifying prognostic comorbidity in longitudinal studies: development and validation. J Chronic Dis. 1987; 40(5):373-83.

51 Verbrugge LM, Lepkowski JM, Imanaka Y. Comorbidity and its impact on disability. Milbank Q. 1989;67(3-4):450-84.
52 Ettinger WH, Davis MA, Neuhaus JM, Mallon KP. Long-term physical functioning in persons with knee osteoarthritis from NHANES. I: effects of comorbid medical conditions. J Clin Epidemiol. 1994 Jul;47(7):80915.

53 Tibshirani R. Regression shrinkage and selection via the lasso. J Roy Stat Soc B Met. 1996; 58(1):267-88.

54 Giaquinto S. Comorbidity in post-stroke rehabilitation. Eur J Neurol. 2003 May;10(3): 235-8.

55 Mulder T, Hochstenbach J. Adaptability and flexibility of the human motor system: implications for neurological rehabilitation. Neural Plast. 2001;8(1-2):131-40.

56 Koroukian SM, Warner DF, Owusu C, Given CW. Multimorbidity redefined: prospective health outcomes and the cumulative effect of co-occurring conditions. Prev Chronic Dis. 2015 Apr;12:E55

57 Kwon S, Hartzema AG, Duncan PW, Min-Lai S. Disability measures in stroke: relationship among the barthel index, the functional independence measure, and the modified rankin scale. Stroke. 2004 Apr;35(4):918-23.

58 Kasner SE. Clinical interpretation and use of stroke scales. Lancet Neurol. 2006 Jul;5(7): 603-12. 